\title{
Abstract from Current Literature
}

\section{Three Cases of Primary Orbital Lymphoblastic Lymphoma}

Md.Abdul Khaleque ${ }^{1}$, Md. Golam Hafiz², Cristian German Sanchez La Rosa ${ }^{3}$, Armando Pena Hernandez ${ }^{4}$,

1,2 Department of Pediatric Hematology and Oncology,Bangabandhu Sheikh Mujib Medical University Dhaka, Bangladesh, ${ }^{3}$ Hospital JP Garrahan Buenos Aires, Argentina, ${ }^{4}$ Hospital Escuela Tegucigalpa , Francisco Morazán, Honduras.

Clinical Findings : We report the cases of three children from different countries (Bangladesh, Honduras, and Argentina) and settings who each presented with a rapidly enlarging orbital mass. They were ultimately given rare diagnoses of primary orbital lymphoblastic lymphoma, and we present them together to highlight the clinical features and the differential diagnosis.

The children ranged in age from three to fifteen years and were previously healthy. They had histories of two to six months of orbital swelling before diagnosis. In two of the cases, the initial diagnosis was an infectious condition which had progressed despite antibiotic therapy. In the other case, the initial diagnosis was inflammatory pseudotumor; however, since the patient had a rapidly enlarging mass, an international referral center was consulted on the biopsy and they confirmed the diagnosis of orbital lymphoblastic lymphoma. In all cases, imaging studies (including orbital CT scan) were done, revealing orbital masses for which incisional biopsy was indicated.

Even though all three children were seen in tertiary care centers, orbital lymphoblastic lymphoma was not initially considered in the differential diagnosis. Therefore, final diagnosis was not made until they had very advanced disease. In all three cases: the pathology report established lymphoblastic lymphoma with T-cell immunophenotype, no other tumor manifestations were evident, including in the bone marrow and the cerebrospinal fluid, the patients were treated with chemotherapy based on protocols for acute lymphoblastic leukemia phenotype, each achieved a complete remission with a follow-up range of three to fourteen months
Discussion: Lymphoblastic lymphoma involving, exclusively, the orbit is extremely rare and only single case reports seem to be published. In systemicallydisseminated leukemia and lymphoma, orbital involvement is rather more common: for instance, the orbit is a typical site for a granulocytic sarcoma related to acute myeloid leukemia (AML) with the $t(8 ; 21)$ translocation. The T-cell phenotype is the most common feature in lymphoblastic lymphoma, but it usually presents with disseminated disease and massive mediastinal masses or hyperleukocytosis. However, localized T-cell lymphoblastic lymphoma in many other extranodal locations has been reported. In localized lymphomas, the B-cell precursor phenotype may be seen with some frequency, especially in the bones, but involvement of the orbit is still rare.

As in other pediatric lymphomas, therapy should be based on histology, immunophenotype and staging. Surgical procedures to resect the tumor mass or radiotherapy play no role in these lymphomas. The orbit may also be a site of relapse.

Orbital lymphomas in adults are much more common and typically include marginal zone lymphomas of MALT (mucosal associated) type. The identification of this malignancy is important since treatment is specific.

In rare cases like the ones presented here, it is essential to procure fresh biopsy material from the tumor in order to perform a full panel immunophenotype by flow cytometry. If this had been done with these three patients, the correct diagnoses could have been made within hours.

Published on Oncopedia: $31^{\text {st }}$ October 2014, online journal of St.Jude children's research hospital, USA.

URL:https://www.cure4kids.org/ums/oncopedia/ case_detail/?id=618

\section{Head growth in very low birth weight premature predicts later cognition}

Fisher PG.

The Journal of Pediatrics, 2014, Volume 165, Issue 6, Pages 1073-1075

Sammallahti et al conducted a cohort study of 103 former very low birth weight preterm infants now at mean age 25 years to distinguish whether faster growth 
from birth to term age and during the first year thereafter predicted neurocognitive abilities. After adjustment for confounding neonatal complications, the investigators found that faster growth from birth to term in head circumference, but not weight or length, predicted intelligence quotient in young adulthood. Growth in weight, length, and head circumference from term to age 12 months did not predict cognitive outcome. The first weeks after birth may well be critical for brain development. This study points once again to the importance of following head circumference for premature infants. Measurement of occipito frontal circumference in the intensive care nursery may add predictive neurocognitive outcome data for both physicians and parents.

\section{Valganciclovir for Symptomatic Congenital Cytomegalovirus Diseasea}

Kimberlin DW, Jester PM, Sánchez PJ, Ahmed A, Arav-Boger R, Michaels MG et al.

National Institute of Allergy and Infectious Diseases Collaborative Antiviral Study Group

N Engl J Med 2015; 372:933-943 March 5, 2015DOI: 10.1056/NEJMoa1404599

Background: The treatment of symptomatic congenital cytomegalovirus (CMV) disease with intravenous ganciclovir for 6 weeks has been shown to improve audiology outcomes at 6 months, but the benefits wane over time.

Methods: We conducted a randomized, placebocontrolled trial of valganciclovir therapy in neonates with symptomatic congenital CMV disease, comparing 6 months of therapy with 6 weeks of therapy. The primary end point was the change in hearing in the better ear ("best-ear" hearing) from baseline to 6 months. Secondary end points included the change in hearing from baseline to follow-up at 12 and 24 months and neurodevelopmental outcomes, with each end point adjusted for central nervous system involvement at baseline.

Results: A total of 96 neonates underwent randomization, of which 86 had follow-up data at 6 months that could be evaluated. Best-ear hearing at 6 months was similar in the 6-month group and the 6week group ( 2 and 3 participants, respectively, had improvement; 36 and 37 had no change; and 5 and 3 had worsening; $P=0.41$ ). Total-ear hearing (hearing in one or both ears that could be evaluated) was more likely to be improved or to remain normal at 12 months in the 6-month group than in the 6-week group (73\% vs. $57 \%, P=0.01)$. The benefit in total-ear hearing was maintained at 24 months ( $77 \%$ vs. $64 \%, P=0.04)$. At 24 months, the 6-month group, as compared with the 6-week group, had better neurodevelopmental scores on the Bayley Scales of Infant and Toddler Development, third edition, on the language-composite component $(P=0.004)$ and on the receptivecommunication scale $(P=0.003)$. Grade 3 or 4 neutropenia occurred in $19 \%$ of the participants during the first 6 weeks. During the next 4.5 months of the study, grade 3 or 4 neutropenia occurred in $21 \%$ of the participants in the 6-month group and in $27 \%$ of those in the 6-week group $(\mathrm{P}=0.64)$.

Conclusions : Treating symptomatic congenital CMV disease with valganciclovir for 6 months, as compared with 6 weeks, did not improve hearing in the short term but appeared to improve hearing and developmental outcomes modestly in the longer term. (Funded by the National Institute of Allergy and Infectious Diseases; ClinicalTrials.gov number, NCT00466817.)

Pediatrics Volume 135, number 4, April 2015

\section{Etiology of Childhood Bacteremia and Timely Antibiotics Administration in the Emergency Department}

Irwin AD, Drew RJ, Marshall P, Nguyen K, Hoyle E, Macfarlane KA et al.

Background: Bacteremia is now an uncommon presentation to the children's emergency department (ED) but is abstract associated with significant morbidity and mortality. Its evolving etiology may affect the ability of clinicians to initiate timely, appropriate antimicrobial therapy.

Methods: A retrospective time series analysis of bacteremia was conducted in the Alder Hey Children's Hospital ED between 2001 and 2011. Data on significant comorbidities, time to empirical therapy, and antibiotic susceptibility were recorded.

Results: A total of 575 clinical episodes were identified, and Streptococcus pneumoniae $(n=109)$, Neisseria meningitidis ( $n=96)$, and Staphylococcus aureus ( $n$ $=89$ ) were commonly isolated. The rate of bacteremia was 1.42 per 1000 ED attendances (95\% confidence 
interval: 1.31-1.53). There was an annual reduction of $10.6 \%$ (6.6\%-14.5\%) in vaccine-preventable infections, and an annual increase of $6.7 \%(1.2 \%-12.5 \%)$ in Gram-negative infections. The pneumococcal conjugate vaccine was associated with a 49\% (32\%$74 \%$ ) reduction in pneumococcal bacteremia. The rate of health care-associated bacteremia increased from 0.17 to 0.43 per $1000 \mathrm{ED}$ attendances $(P=.002)$. Susceptibility to empirical antibiotics was reduced (96.3\%-82.6\%; P , .001). Health care-associated bacteremia was associated with an increased length of stay of 3.9 days (95\% confidence interval: $2.3-$
5.8). Median time to antibiotics was 184 minutes (interquartile range: 63-331) and 57 (interquartile range: 27-97) minutes longer in Gram-negative bacteremia than in vaccine-preventable bacteremia.

Conclusions: Changes in the etiology of pediatric bacteremia have implications for prompt, appropriate empirical treatment. Increasingly, pediatric bacteremia in the ED is health care associated, which increases length of inpatient stay. Prompt, effective antimicrobial administration requires new tools to improve recognition, in addition to continued etiological surveillance 